\title{
Antibiofilm Effect of Cerium Nitrate against Bacteria and Yeast
}

\author{
Azevedo MM ${ }^{1 *}$, Cobrado $\mathrm{L}^{1,2,3}$, Silva Dias $\mathrm{A}^{1,2}$, Ramalho $\mathrm{P}^{1}$, Pina-Vaz $\mathrm{C}^{1,2,4}$ and Rodrigues $\mathrm{AG}^{1,2,3}$ \\ ${ }^{1}$ Department of Microbiology, Faculty of Medicine, University of Porto, Portugal \\ ${ }^{2}$ Cardiovascular Research \& Development Unit, Faculty of Medicine, University of Porto, Portugal \\ ${ }^{3}$ Burn Unit and Department of Plastic and Reconstructive Surgery, Hospital S. Jo ao, Portugal \\ ${ }^{4}$ Department of Microbiology, Hospital S. Jo ao, Portugal
}

Received: November 07, 2013; Accepted: December 24, 2013; Published: December 27, 2013

*Corresponding author: Azevedo MM , Faculdade de Medicina da Universidade do Porto, Portugal, Tel: 351-913-235-755; Fax: 351-225-513-662; Email: maria.manuel.azevedo2011@gmail.com

\section{Abstract}

Staphylococcus aureus, Staphylococcus epidermidis, Acinetobacter baumannii and Candida albicans are frequent agents of catheterrelated bloodstream infections, which increase the time of hospitalization and related medical costs. In order to find new strategies to reduce the colonization of these organisms on indwelling medical devices, this research aimed to determine the antibiofilm effect of cerium nitrate.

XTT and crystal violet assays were used to quantify biofilms challenged with cerium nitrate. Two strains of Staphylococcus aureus, Staphylococcus epidermidis, Acinetobacter baumannii and Candida albicans were used, grown on polystyrene surfaces.

Concerning the biofilm metabolic activity of Staphylococcus epidermidis, all the tested concentrations of cerium nitrate inhibited significantly both strains, however for Staphylococcus aureus only the highest concentration tested inhibited significantly both strains. Referring to the total biomass, cerium nitrate concentrations of $3.2 \times 10^{2}$ or higher inhibited significantly both strains of Staphylococcus aureus and Staphylococcus epidermidis, however only the higher concentration tested inhibited the total biomass of Acinetobacter baumannii.

Concerning the biofilm metabolic activity of $C$. albicans, cerium nitrate at concentrations of $6.4 \times 10^{2} \mathrm{mg} / \mathrm{L}$ inhibited significantly both strains. Referring to the total biomass, cerium nitrate at a considerably lower concentration $\left(0.8 \times 10^{2} \mathrm{mg} / \mathrm{L}\right)$ inhibited both strains of $C$. albicans.

The fact that cerium nitrate inhibited more effectively the total biomass production than the metabolic activity in these strains (except for Staphylococcus epidermidis), may be explained by interference with extracellular matrix production or with intercellular communication. Whichever might be the case, cerium nitrate has a potent antibiofilm effect upon these strains and deserves further attention in strategies that could lower microbial colonization of indwelling medical devices.

Keywords: Catheter-related bloodstream infection; Central venous catheters; Staphylococcus aureus; Staphylococcus epidermidis; Acinetobacterbaumannii, C. albicans. Biofilms; Cerium nitrate.

\section{Introduction}

Staphylococcus aureus, S. epidermidis, Acinetobacter baumannii and Candida species are among the most frequently isolated microorganisms from central venous catheters (CVCs) placed in critically ill patients [1]. Staphylococci are capable of forming biofilms on implanted devices, causing infections [2]. The emergence of $S$. epidermidis as a pathogen is also linked to the extensive use of catheters. Their capacity to origin infection is mainly related to the ability to form mucoid biofilms on the inert synthetic surfaces of indwelling medical devices [3]. Among bloodstream infections caused by Candida species, C. albicans is the most frequent isolate, being associated with a high mortality rate. As a whole, C. albicans stands on as the fourth leading cause of catheter-related bloodstream infections (CRBSIs) [1,4], which increase the time of hospitalization and related medical costs $[5,6]$.

Data from literature [7], show that over $65 \%$ of hospital infections are originated from biofilm forming organisms. A major concern when biofilms develop is the emergence of microbial strains resistant to the host immune system and to antimicrobial therapy [2]. A correlation between Candida spp. biofilm formation and resistance to antimicrobial agents was already documented P.K. Mukherjee, et al. [8]. Despite antifungal therapy, the persistence of fungi was noticed.

Therefore, new agents that could inhibit biofilm formation would be highly desirable. Cerium is a rare earth element of the lanthanide group and cerium nitrate is currently used in the management of burn wounds, with a reduction in patient morbidity and mortality [9]. Cerium nitrate was reported to reduce the activity of microbial pathogens due to their action at different cellular targets [10]. This compound might be quite useful in medical applications because toxicity is rare with lanthanides and in addition is an inexpensive compound.

The aim of this research was to determine the antibiofilm effect of cerium nitrate upon Staphylococcus aureus, Staphylococcus 
epidermidis, Acinetobacter baumannii and Candida albicans, frequent microbial colonizer's of indwelling medical devices like central venous catheters (CVCs).

\section{Materials and Methods}

\section{Microbial strains and chemicals}

A type strain and a clinical isolate of each of four microbial species were used. The type strains from the American type Culture Collections were S. aureus ATCC 29213, S. epidermidis ATCC 155, A. baumanii ATCC 19606 and C. albicans ATCC 90028; the clinical strains of $S$. aureus (SA1), S. epidermidis (SE1), A. baumanii (AB1) and $C$. albicans (CA1) had been previously isolated from cultures of CVCs removed from critical care patients admitted at Hospital S. Joao (Porto, Portugal). All clinical isolates had been identified by Vitek System (bioMerieux, Vercieux, France).

Bacterial strains were kept frozen in Luria-Bertani broth (LB) (Difco Laboratories, Detroit, MI, USA) supplemented with 20\% glycerol, and the yeast strains in yeast potato dextrose medium (YPD) (Difco Laboratories) supplemented with $40 \%$ glycerol at $-70^{\circ} \mathrm{C}$ until testing. For each experiment, the microorganisms were subcultured twice on LB agar, $37^{\circ} \mathrm{C}, 24 \mathrm{~h}$ (bacteria) or Sabouraud agar $35^{\circ} \mathrm{C}, 24 \mathrm{~h}$ (Difco Laboratories) (yeasts) to assess the purity of the culture and its viability.

The chemical used in the experiments was cerium nitrate cerium (III) nitrate hexahydrate, Sigma-Aldrich.

\section{Biofilm formation}

Inoculum preparation: To prepare bacterial inoculum's, one colony of each bacterial isolate was used to inoculate $5 \mathrm{ml}$ of LB medium. The cultures were incubated during $18 \mathrm{~h}$ at $37^{\circ} \mathrm{C}$, at $180 \mathrm{rpm}$. Following this period the number of cells in each culture was adjusted to 0.5 MacFarland density in LB medium and subsequent diluted 1:10 in order to obtain $10^{7}$ cells.

Yeast cells were grown for $18 \mathrm{~h}$ in Sabouraud broth at $35^{\circ} \mathrm{C}$, at $180 \mathrm{rpm}$. Afterwards, cells were washed in PBS, ressuspended in RPMI and adjusted to $0.5 \mathrm{McF}$ arland density in order to achieve $10^{6}$ cells $/ \mathrm{mL}$.

Biofilm growth with cerium nitrate: The effect upon biofilm formation was tested with four concentrations of cerium nitrate $\left(6.4 \times 10^{2} \mathrm{mg} / \mathrm{L}, 3.2 \times 10^{2} \mathrm{mg} / \mathrm{L}, 1.6 \times 10^{2} \mathrm{mg} / \mathrm{L}\right.$ and $0.8 \times 10^{2}$ $\mathrm{mg} / \mathrm{L})$, which were selected based on the results described by L. Cobrado \& al. [2]. Each concentration was added to 12-well polystyrene microtiter plates containing the inoculum in order to obtain a final volume of $1 \mathrm{ml}$. In order to obtain biofilm formation, all the strains were incubated for $24 \mathrm{~h}$ at $37^{\circ} \mathrm{C}$.

\section{Biofilm quantification}

Metabolic activity of biofilm cells - XTT assay: A XTT reduction assay was used to determine the in situ biofilm metabolic activity. After biofilm formation, supernatants were removed from the plates and $1 \mathrm{ml}$ of PBS buffer was added and gently mixed. Following PBS rejection, $1 \mathrm{ml}$ of XTT [2,3-bis (2methoxy4-nitro-5sulfophenyl)-2H-tetrazolium-5-carboxanilide] solution was added to each prewashed biofilm and control wells. Plates were further incubated for $5 \mathrm{~h}$ at $37^{\circ} \mathrm{C}$, in the dark, and finally the optical density (OD) was measured at $492 \mathrm{~nm}$. The XTT solution was prepared in PBS (4 mg XTT in $10 \mathrm{ml}$ prewarmed at $37^{\circ} \mathrm{C}$ ); this solution was supplemented with menadione prepared in acetone. All the assays were performed in triplicate.

Biomass formation of biofilm cells - crystal violet assay: Biofilm fixation was performed with $1 \mathrm{ml} 99 \%$ methanol (15 $\mathrm{min})$, after which supernatants were removed and the plates airdried. Afterwards, $1 \mathrm{ml}$ of a crystal violet (CV) solution $(0.02 \%$; $\mathrm{v} / \mathrm{v}$ ) was added to each well; following $20 \mathrm{~min}$, the excess of CV was removed by washing the plates twice with distilled water. Finally, bound CV was released by adding $1.5 \mathrm{ml}$ of acetic acid (33\%; v/v). The optical density (OD) was measured at $590 \mathrm{~nm}$. All the assays were performed in triplicate.

\section{Data analysis}

Biofilm metabolic activity and total biomass with cerium nitrate were expressed as the percentage in relation to the control. Values were divided by 1000 and arcsine square root transformed to achieve normal distribution and homoscedasticity [11].

For each concentration and each strain, biofilm formation was compared by one-way ANOVA, followed by a Dunnett's test to identify significant effects. $P$ value $<0.05$ was considered significant.

\section{Results}

\section{Biofilm formation on polystyrene plates}

Concerning the biofilm metabolic activity of C. albicans, cerium nitrate at $6.4 \times 10^{2} \mathrm{mg} / \mathrm{L}$ inhibited significantly the clinical strain by $36 \%( \pm 12)$ and the ATCC strain by $28 \%( \pm 4)$ (Figure 1A).

Referring to the total biomass of $C$. albicans, cerium nitrate at $0.8 \times 10^{2} \mathrm{mg} / \mathrm{L}$ inhibited significantly the clinical strain by $81 \%$ $( \pm 11)$ and the ATCC strain by $50 \%( \pm 4)$ (Figure $1 \mathrm{~B}$ ). Doubling the concentration of cerium nitrate to $1.6 \times 10^{2} \mathrm{mg} / \mathrm{L}$, there was a reduction in the total biomass of the clinical strain by $87 \%( \pm 11)$ and of the ATCC strain by $80 \%( \pm 5)$ (Figure 1B).

No relevant biofilm metabolic activity inhibition was found for $S$. aureus with cerium nitrate at concentrations $\leq$ than $\left(3.2 \times 10^{2} \mathrm{mg} / \mathrm{L}\right)$ (Figure $\left.2 \mathrm{~A}\right)$, however an inhibition in total biomass was found for concentrations $\geq 3.2 \times 10^{2} \mathrm{mg} / \mathrm{L}(\mathrm{p}<0.01)$ (Figure 2B).

Cerium nitrate at all the tested concentrations reduced significantly $S$. epidermidis biofilm metabolic activity $(\mathrm{p}<0.01)$ [Figure $3 \mathrm{~A}$ ], and concentrations $\geq 3.2 \times 10^{2} \mathrm{mg} / \mathrm{L}$ significantly inhibited total biomass $(\mathrm{p}<0.05)$ [Figure 3B].

In respect of $A$. baumannii none of the tested concentrations inhibited biofilm metabolic activity (data not shown), nevertheless the higher tested concentration inhibited total biomass $21 \pm 0 \%$ (Figure $4 \mathrm{~A}$ ). 


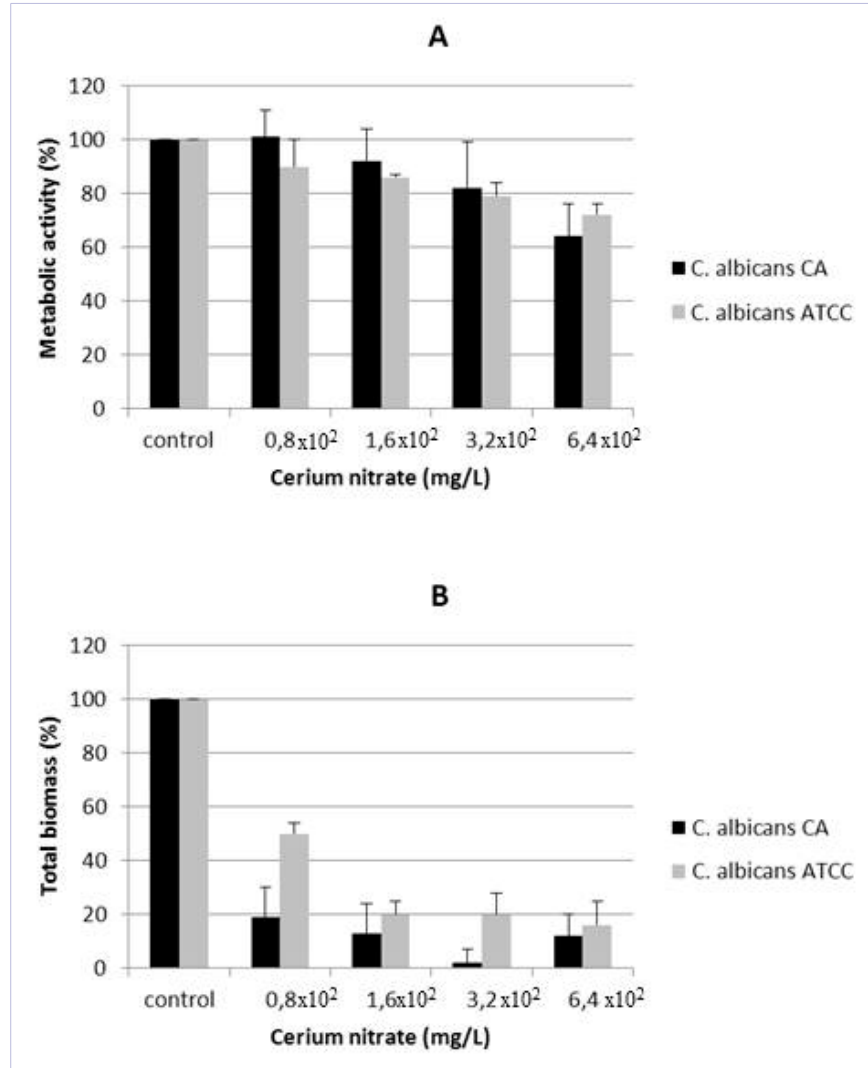

Figure 1: Effect of cerium nitrate on metabolic activity (A) and total biomass (B) (as a percentage of the control) by Candida albicans clinical strain (CA) and ATCC strain. ${ }^{*}$ ) P value $<0.05$.

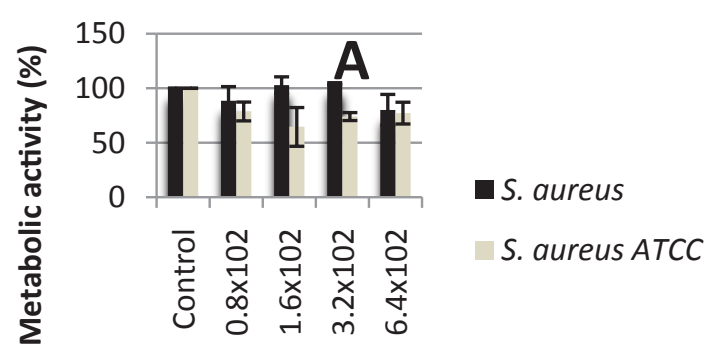

Cerium nitrate $(\mathrm{mg} / \mathrm{L})$

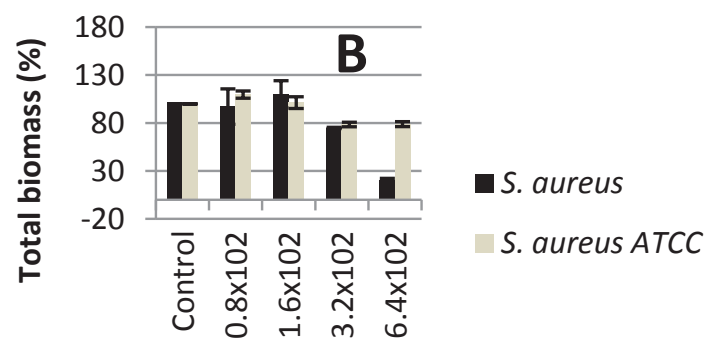

Cerium nitrate $(\mathrm{mg} / \mathrm{L})$

Figure 2: Effect of cerium nitrate on metabolic activity (A) and total biomass (B) (as a percentage of the control) by $S$. aureus clinical strain (SA1) and ATCC strain. (*) P value $<0.05$.

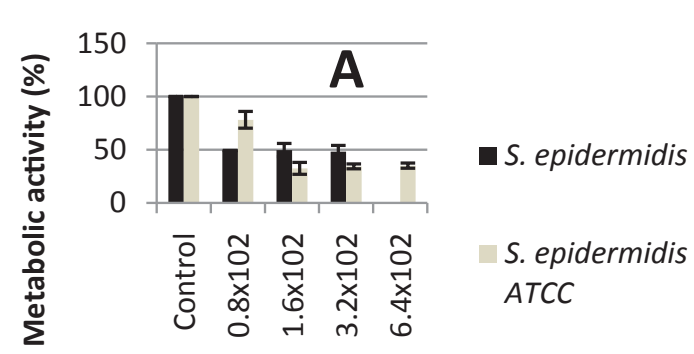

Cerium nitrate $(\mathrm{mg} / \mathrm{L})$

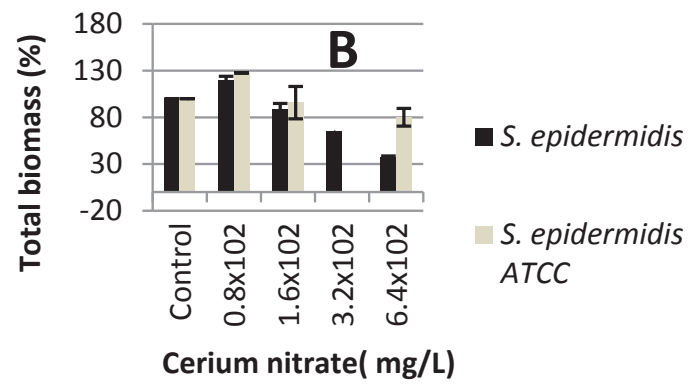

Figure 3: Effect of cerium nitrate on metabolic activity (A) and total biomass (B) (as a percentage of the control) by S. epidermidis clinical strain (SE1) and ATCC strain. $\left({ }^{*}\right)$ P value $<0.05$.

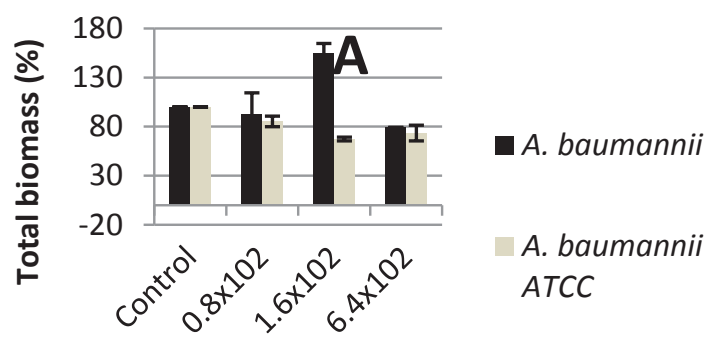

Cerium nitrate $(\mathrm{mg} / \mathrm{L})$

Figure 4: Effect of cerium nitrate on total biomass (A) (as a percentage of the control) by A. baumannii clinical strain (AB1) and ATCC strain. $(*)$ P value $<0.05$.

\section{Discussion}

The frequent use of indwelling devices in medical care makes patients more prone to develop biofilm-related infections, with a negative impact on the clinical outcome and hospital costs. The ability of nosocomial pathogens such as $C$. albicans to form biofilms is clinically relevant, since their formation impairs the efficacy of antimicrobial therapy [12]. Therefore, it is crucial to find new modes of prevention and effective alternatives to antimicrobial treatment.

Cerium nitrate is thought to enter the cytoplasm of microorganisms and inhibit cellular respiration, glucose metabolism and, eventually, induce disruption of the cell membrane (Dwight and Johnson 1967). According to the 
results of J.P. Garner \& et al.[9], cerium nitrate exhibits a bacteriostactic effect, but further testing demonstrated a microbicidal effect against a wide range of microbial pathogens [13]. Moreover, a potent antibiofilm activity against $C$. albicans grown on polyurethane catheter segments was documented with concentrations lower than MIC: cerium nitrate, at $1.7 \times 10^{3} \mathrm{mg} / \mathrm{L}$, inhibited biofilm formation by $\sim 60 \%$. The results were similar either at $24 \mathrm{~h}$ or $48 \mathrm{~h}$ of incubation.

The present study confirms the antibiofilm effect of cerium nitrate against $C$. albicans even at lower concentrations. This work also reveal that cerium nitrate at all the tested concentrations inhibited significantly $S$. epidermidis biofilm metabolic activity, however only concentrations $\geq 3.2 \times 10^{2} \mathrm{mg} / \mathrm{L}$ significantly inhibited total biomass $[14,15]$. The effect of cerium nitrate on biofilm inhibition is specie and concentration dependent. Despite both XTT and CV assays were reproducible, as estimated by the low values of standard deviation, major differences in the quantification of the biofilm were observed. The XTT assay is based on the reduction of tetrazolium salts by mitochondrial dehydrogenases of yeasts and it is used for the quantification of metabolically active cells. The less expensive and timeconsuming CV assay is commonly used for the quantification of biofilm biomass, staining the cells (living and dead) and the matrix produced [3]. The fact that cerium nitrate inhibited more effectively the total biomass production than the metabolic activity of the yeast cells, $S$. aureus and A. baumannii strains, may be explained by its interference with intercellular communication or with extracellular matrix production. Whichever might be the case, further studies are being held to clarify the mechanism of action and the endovascular biocompatibility of cerium nitrate, in order to support the clinical use of such promising compound to coat indwelling medical devices.

\section{Conclusion}

Nowadays medical device colonization constitutes a global threat that may contribute to antimicrobial resistance. The present study clearly demonstrated the antibiofilm effect of cerium nitrate against bacteria and yeast strains. Further studies are being conducted in order to clarify the mechanism of biofilm inhibition and the biocompatibility of cerium nitrate, which may play a promising role in biomedical applications in the future.

\section{References}

1. Cobrado L, Espinar MJ, Costa-de-Oliveira S, Silva AT, Pina-Vaz C, et al
(2010) Colonization Of Central Venous Catheters In Intensive Care Patients: A 1-Year Survey In A Portuguese University Hospital. Am. J. Infect. Control, 38(1): 83-84.

2. Gov Y, Borovok I, Korem M, Singh VK, Jayaswal RK, Wilkinson BJ, et al. (2004) Quorum Sensing In Staphylococci Is Regulated Via Phosphorylation Of Three Conserved Histidine Residues. J Biol Chem, 279(15): 14665-14672.

3. O Gara JP, Humphreys H (2001) Staphylococcus Epidermidis Biofilms: Importance And Implications. J. Med. Microbiol, 50(7): 582-587.

4. Orsi GB, Di Stefano L, Noah N (2002) Hospital-Acquired, LaboratoryConfirmed Bloodstream Infection: Increased Hospital Stay And Direct Costs. Infect. Control Hosp. Epidemiol, 23(4)L: 190-197.

5. Mukherjee PK, Chandra J (2004) Candida Biofilm Resistance. Drug Resistance Updates, 7(4): 301-309.

6. Peeters E, Nelis HJ, Coenye T (2008) Comparison Of Multiple Methods For Quantification Of Microbial Biofilms Grown In Microtiter Plates. J. Microbiol. Methods, 72(2): 157-165.

7. Kiran MD, Adikesavan NV, Cirioni O, Giacometti A, Silvestri C, Scalise G, et al. (2008) Discovery of a quorum-sensing inhibitor of drug-resistant staphylococcal infections by structure-based virtual screening. Mol. Pharmacol, 73(5): 1578-1586.

8. Mah TF, O Toole GA (2001) Mechamisms Of Biofilm Resistance To Antimicrobial Agents. Trends Microbiol, 9(1): 34-39.

9. Garner J P, Heppell P S (2005) Cerium Nitrate In The Management Of Burns. Burns., 31(5): 539-547.

10. Dwight ET, Johnson GT (1967) Some Effects Of Rare Earth Elements And Yttrium On Microbial Growth. Mycologia, 59: 492-503.

11. Polderman KH, Girbes AR (2002) Central Venous Catheter Use. Part 2: Infectious Complications. Intensive Care Med, 28(1):18-28.

12. Donlan RM, Costerton JW (2002) Biofilms: Survival Mechanisms Of Clinically Relevant Microorganisms. Clin. Microbiol. Review, 15(2): 167-193.

13. Cobrado L, Azevedo MM, Silva-Dias A, Ramos JP, Pina-Vaz C, Rodrigues AG (2012) Rodrigues, Cerium, Chitosan And Hamamelitannin As Novel Biofilm Inhibitors? J. Antimicrob. Chemother, 67(5): 1159-1162.

14. Tamura NK, Gasparetto A, Svidzinski TI (2003) Evaluation Of The Adherence Of Candida Species To Urinary Catheters. Mycopathologia, 156(4): 269-272.

15. Zar JH (1996) Biostatistical Analysis. (3rd Ed.), New Jersey: PrenticeHall. 